\title{
A case of pediatric anaplastic large cell lymphoma with hemophagocytic lymphohistiocytosis mimicking juvenile myelomonocytic leukemia
}

\author{
Jonathan M. Loree • Marta Rojas-Vasquez • \\ Artur J. Szkotak
}

Received: 30 September 2010 / Accepted: 5 January 2011 /Published online: 27 January 2011

(C) Springer-Verlag 2011

\begin{abstract}
Hemophagocytic lymphohistiocytosis (HLH) is a life-threatening inflammatory syndrome characterized by reactive proliferation of histiocytes in hematopoietic tissues, with accompanying hemophagocytosis. A number of primary and secondary triggers have been identified, including some hematolymphoid neoplasms. We submit the case of an 11-year-old female who presented with HLH and met diagnostic criteria for juvenile myelomonocytic leukemia, which included the apparently non-recurrent clonal cytogenetic abnormality $\mathrm{t}(5 ; 12)(\mathrm{q} 35 ; \mathrm{q} 24.1)$. The diagnosis was eventually revised to systemic anaplastic large cell lymphoma with cryptic NPM-ALK fusion. We review the previously reported cases of pediatric HLH triggered by systemic ALCL and find that the marked neutrophilia, monocytosis, and circulating myeloid precursors seen in our case were unique. In addition, our review highlights the challenge of detecting ALCL in the context of HLH, and identifies features that may be of assistance. While the bone marrow is the most frequently examined tissue in HLH, its diagnostic yield is low. The suspicion for ALCL, and perhaps other neoplasia, should be higher when
\end{abstract}

\section{J. M. Loree}

Faculty of Medicine and Dentistry,

University of Alberta,

Edmonton, AB T6G 2B7, Canada

\section{Rojas-Vasquez}

Department of Pediatrics, Walter Mackenzie Centre,

University of Alberta,

Edmonton, AB T6G 2B7, Canada

\section{A. J. Szkotak $(\bowtie)$}

Department of Laboratory Medicine and Pathology, 4B1.32

Walter Mackenzie Centre, University of Alberta,

Edmonton, AB T6G 2B7, Canada

e-mail: artur.szkotak@albertahealthservices.ca associated with pulmonary disease (especially if multinodular), skin lesions or lymphadenopathy. Under these circumstances, additional biopsies are recommended even when lymph node enlargement is minimal - a finding often attributed directly to HLH and ignored. Furthermore, it is important to assess for both CD30 and ALK expression, because malignant cells may be too sparse to be detectable with routine $\mathrm{H} \& \mathrm{E}$ or $\mathrm{CD} 3$ stains and may not consistently demonstrate both markers.

Keywords Anaplastic large cell lymphoma.

Hemophagocytic lymphohistiocytosis · Bone marrow Multiple pulmonary nodules $\cdot$ Juvenile myelomonocytic leukemia

\section{Introduction}

Hemophagocytic lymphohistiocytosis (HLH) is an acute, life-threatening syndrome that occurs in children and young adults typified by reactive proliferation of histiocytes in the bone marrow, liver, spleen, and lymph nodes with characteristic hemophagocytosis in affected tissues and overwhelming systemic inflammatory response. The underlying cause of the disease can be traced to genetic and/or acquired impairments in natural killer (NK) cell function, or less commonly, decreased NK cell numbers. Through an incompletely understood mechanism this leads to dysregulation of histiocyte, cytotoxic T-cell and type 1 helper T-cell function causing over-secretion of pro-inflammatory cytokines, which further stimulate histiocytic proliferation and activity in a positive feedback loop [1].

Common findings in HLH include fever, hepatosplenomegaly, cytopenias, disseminated intravascular coagulation (DIC) and neurologic symptoms; less frequently there is 
lymphadenopathy, skin rash, diarrhea, jaundice, and edema. These findings are usually accompanied by characteristic changes in biochemical parameters secondary to histiocyte over-stimulation. The most important of these are hypertriglyceridemia of $>3 \mathrm{mmol} / \mathrm{L}$, elevated serum ferritin of $>500 \mathrm{mcg} / \mathrm{L}$, and increased soluble CD25 (IL-2 Receptor). Also of use in the diagnosis of HLH, is a demonstration of decreased NK cell function (or number) as well as examination of tissue samples from bone marrow, liver, spleen, lymph nodes, and/or CSF for excess hemophagocytosis. A diagnostic algorithm on the basis of clinical, biochemical and pathologic findings has been developed and is reviewed elsewhere [2].

HLH is associated with high mortality rates and it is of vital importance to resolution that the underlying cause is identified and treated, where possible. Primary HLH is known to occur with a number of heritable mutations in NK cell genes, including those that encode Perforin (PRF1), MUNC 13-4 $(U N C 13 D)$ and Syntaxin 11 (STX11) [1,2]. Secondary causes of HLH include infection, malignancy, rheumatologic disease, immunodeficiency and drugs/toxins [1-3]. Of the malignancies, T- and NK-cell neoplasms are the most commonly implicated but others have been described [2, 4]. Investigation for hematolymphoid neoplasia is of particular importance because of the availability of effective treatment and because of their progressive nature. However, HLH causes a constellation of multisystem findings, reactive organomegaly and distorted tissue architecture that often makes it difficult to identify or classify the underlying disease.

Systemic ALCL is a mature T-cell neoplasm, which occurs mainly in children and young adults, accounting for $10-15 \%$ of non-Hodgkin lymphomas in pediatric patients [5]. Primary cutaneous ALCL shares some histomorphologic features with systemic ALCL but is considered a distinct disease entity and is not considered further in this manuscript [5]. Systemic ALCL involves lymph nodes, liver, bone marrow, skin, and lung [6]. This disease is frequently associated with potent inflammatory responses resulting in constitutional symptoms, making a causal relationship to HLH mechanistically plausible [7]. Microscopic examination reveals variable morphology with a portion of cells that demonstrate intermediate to very large size, with eccentric horse-shoe or kidney-shaped nuclei containing multiple nucleoli, and may contain an eosinophilic region near the nucleus [8]. These characteristic "hallmark" cells often account for only a small proportion of neoplastic cells - making their identification difficult. The characteristic immunophenotype is surface and Golgi CD30 staining, usually accompanied by loss of one or more T-cell specific markers including CD3. Surface, cytoplasmic and/or nuclear staining with anaplastic lymphoma kinase (ALK) is a very specific finding [8]. Normally, ALK staining is absent in all tissues except for rare cells in the brain, but in systemic ALCL, there are a number of recurrent translocations that fuse $A L K$ to a partner gene. The most common translocation is the $\mathrm{t}(2 ; 5)(\mathrm{p} 23 ; \mathrm{q} 35)$ which fuses $A L K$ on 2 p23 to the nucleophosmin (NPM) gene on $5 \mathrm{q} 35$ and occurs in approximately $85 \%$ of ALK+ ALCL $[9,10]$. There is also a subset of systemic ALCL that is ALK-, which occurs mainly in older adults $[5,6]$.

Juvenile myelomonocytic leukemia (JMML) is a rare myeloid neoplasm characterized by proliferation of cells of the granulocytic and monocytic lineages. It occurs exclusively in those $<13$ years of age and accounts for only 2 $3 \%$ of childhood leukemias $[11,12]$. Though not typically associated with $\mathrm{HLH}$, the simultaneous occurrence of these two disease processes has been recently described [4]. The diagnosis of JMML is challenging under the best of circumstances because of its non-specific pathologic features, but this becomes even more of a challenge when associated with a potent inflammatory condition such as HLH. The WHO diagnostic criteria for JMML are (1) peripheral monocytosis $>1 \times 10^{9} / \mathrm{L}$, (2) blasts $<20 \%$, (3) exclusion of $B C R-A B L 1$, and (4) two or more of: (a) increased $\mathrm{HbF}$, (b) circulating myeloid precursors, (c) WBC $>10 \times 10^{9} / \mathrm{L}$, (d) clonal chromosomal abnormality, or (e) GM-CSF hypersensitivity in vitro [11]. While these criteria work reasonably well in practice, it seems plausible that a reactive condition characterized by rapid proliferation of the monocytic-histiocytic lineage, such as HLH, could conceivably result in false positives.

The diagnosis of a hematolymphoid neoplasm can be very difficult to make in the context of HLH because of overlapping clinical and morphologic features. Furthermore, even when there is good evidence of an underlying neoplasm, such as a clonal cytogenetic abnormality, it can make classifying the disease process difficult because there is uncertainty about which features are attributable to HLH and which are attributable to the underlying malignancy. We report the case of a previously healthy 11-year-old female who presented with HLH and was tentatively diagnosed with JMML on the basis of established criteria, which included an apparently non-recurrent clonal cytogenetic abnormality. Subsequent events led to revision of the diagnosis to ALK + ALCL associated with cryptic NPM$A L K$ fusion. Our aim is to describe how the misleading features of this case led to the wrong diagnosis and to review cases of ALCL presenting with HLH described in the literature in order to highlight characteristics of this disease that can be used to prevent misdiagnosis.

\section{Materials and methods}

Patient information from the presented case was used with full consent of the legal guardian. Bone marrow aspirate 
samples were collected into EDTA anticoagulated Vacutainer tubes (BD Canada, Mississauga, ON) followed immediately by slide preparation at the bedside and air drying. Slides were fixed with absolute methanol and stained with May-Grünwald Giemsa (Richard-Allan Scientific, Kalamazoo, MI) for examination. The trephine biopsy was fixed in B-Plus (BBC Biochemical, Mount Vernon, WA), decalcified with acid-EDTA (Cal-EX, Fisher Scientific, Pittsburgh, PA), paraffin embedded (Paraplast), sectioned at $1-2 \mu \mathrm{m}$ thickness, deparaffinized and rehydrated using xylene and ethyl alcohol. A portion of slides were stained with hematoxylin-eosin (H\&E, Surgipath SelecTech, Leica Microsystems Canada, Winnipeg, MB). Immunostaining was carried out with mouse monoclonal antibodies for CD68 (clone PG-M1, 1:400, Dako, Glostrup, Denmark) using the Dako FLEX detection system after antigen retrieval with Dako High pH Solution, CD30 (clone Ber-H2, 1:40, Dako) using the Ventana (Tucson, AZ) iVIEW detection system after antigen retrieval with Dako High pH Solution and ALK1 (clone ALK-01, prediluted, Ventana) using the Ventana ultraView detection system after online antigen retrieval using $\mathrm{CC} 1$ buffer.

A computerized search of English language literature on Medline was performed using the MeSH terms "Lymphohistiocytosis, Hemophagocytic" and "Lymphoma, LargeCell, Anaplastic", further limited to pediatric patients. This approach resulted in the identification of seven publications describing eight relevant cases [13-19]. Seven of these cases met the full diagnostic criteria for HLH (HLH-2004) [2]; one of the cases described by Sevilla et al. only met four HLH criteria and will not be considered further [14]. All discussed cases had biopsy-proven ALCL. Clinical, pathologic, and molecular characteristics were tabulated and compared from each case.

\section{Case report}

An 11-year-old female patient of Filipino descent presented with headache, sore throat, cough, and fever of $40^{\circ} \mathrm{C}$ in late 2009. She was treated initially with amoxicillin and acetaminophen without any response and presented again 8 days later in respiratory distress, requiring intubation and ventilator support. Chest X-rays demonstrated bilateral lower lobe patchy airspace disease, pleural effusions, and interstitial opacification. After 10 days, the patient was extubated but rapidly deteriorated with acute respiratory distress syndrome, hypotension, severe lactic acidosis, renal failure, and liver insufficiency. The findings on chest X-ray were similar to that previously described, but, in addition, fine nodular opacities were also present throughout both lungs. She required repeat intubation on the same day that she was extubated and was progressed from conventional ventilation to high frequency oscillatory ventilation. A nasal swab performed at this time was positive for influenza A of type H1, however the sample was inadequate to further differentiate whether this was H1N1 serotype, the predominate strain in the community at that time.

The patient required aggressive therapy with inotrope support, Prisma hemofiltration, as well as multi-agent antibiotic and antiviral therapy. While in intensive care, she developed anemia $(\mathrm{Hgb}=88 \mathrm{~g} / \mathrm{L}$ initially, but quickly deteriorated and required transfusion support), thrombocytopenia $\left(\right.$ Platelets $\left.=41 \times 10^{9} / \mathrm{L}\right)$, leukocytosis $(\mathrm{WBC}=$ $\left.78.0 \times 10^{9} / \mathrm{L}\right)$, elevated LDH $(1,016 \mathrm{IU} / \mathrm{L})$, elevated ferritin $(10,290 \mathrm{mcg} / \mathrm{L})$, hypertriglyceridemia $(3.76 \mathrm{mmol} / \mathrm{L})$, and hypofibrinogenemia $(0.8 \mathrm{~g} / \mathrm{L})$. On the peripheral blood smear, there was a marked neutrophilia $\left(58.5 \times 10^{9} / \mathrm{L}\right)$ with hypergranulation, Döhle bodies and vacuolation, as well as circulating myeloid precursors $\left(1.6 \times 10^{9} / \mathrm{L}\right)$. Circulating NRBCs $(2 / 100$ WBC) were also present but blast cells were not identified. Lymphocytes were normal in number $\left(6.2 \times 10^{9} / \mathrm{L}\right)$ and morphology. There was a marked monocytosis $\left(11.7 \times 10^{9} / \mathrm{L}\right)$ present with a minority of forms demonstrating atypical morphology including convoluted nuclear shape, increased nuclear:cytoplasmic ratio or deep cytoplasmic basophilia; all forms demonstrated mature chromatin texture. These findings were accompanied by increasing abdominal girth (CT imaging studies demonstrated a normal liver but moderate ascites), marked splenomegaly $(17.5 \mathrm{~cm})$ with a heterogeneous character, and numerous mildly enlarged retroperitoneal and mesenteric lymph nodes (largest was $1.4 \times 1.8 \mathrm{~cm}$ ). Together, these findings were highly suspicious for HLH, prompting bone marrow examination.

The bone marrow aspirate sample is cellular and particulate, with histiocytic proliferation and striking hemophagocytosis of both mature erythrocytes and leukocytes, as well as their progenitors (Fig. 1a). There is also a marked marrow monocytosis comprising $24 \%$ of nucleated cells; these demonstrate mature nuclear chromatin. Blasts account for $3 \%$ of nucleated cells and are not present in excess. The trephine biopsy demonstrates approximately 80-90\% cellularity (Fig. 1b), with diffuse histiocytic hyperplasia (Fig. 1c). These histiocytes demonstrate copious eosinophilic cytoplasm with vacuolation and hint at hemophagocytosis, though this cannot be confirmed on $\mathrm{H} \& \mathrm{E}$ alone as hematopoietic cells cannot definitively be localized within them (Fig. 1c). However, immunostaining of the histiocyte cytoplasm with CD68 PG-M1 antibody confirms their intracellular location (Fig. 1d). There is no evidence of lymphoid aggregate formation, with scarce CD20+ B-cells and a normal CD3 + contingent of diffusely distributed T-cells. The CD4:CD8 ratio appears approximately normal (2:1). The diagnosis of HLH was confirmed on the basis of these findings and presumed to be secondary 
Fig. 1 Bone marrow aspirate and trephine biopsy demonstrating hemophagocytosis. a Aspirate demonstrating hemophagocytosis (May-Grünwald Giemsa). Trephine biopsy at medium power $\mathbf{b}$ and at high power $\mathbf{c}$ demonstrating diffuse histiocytosis. d Histiocyte cytoplasmic immunostaining with CD68 PG-M1 highlights hemophagocytosis
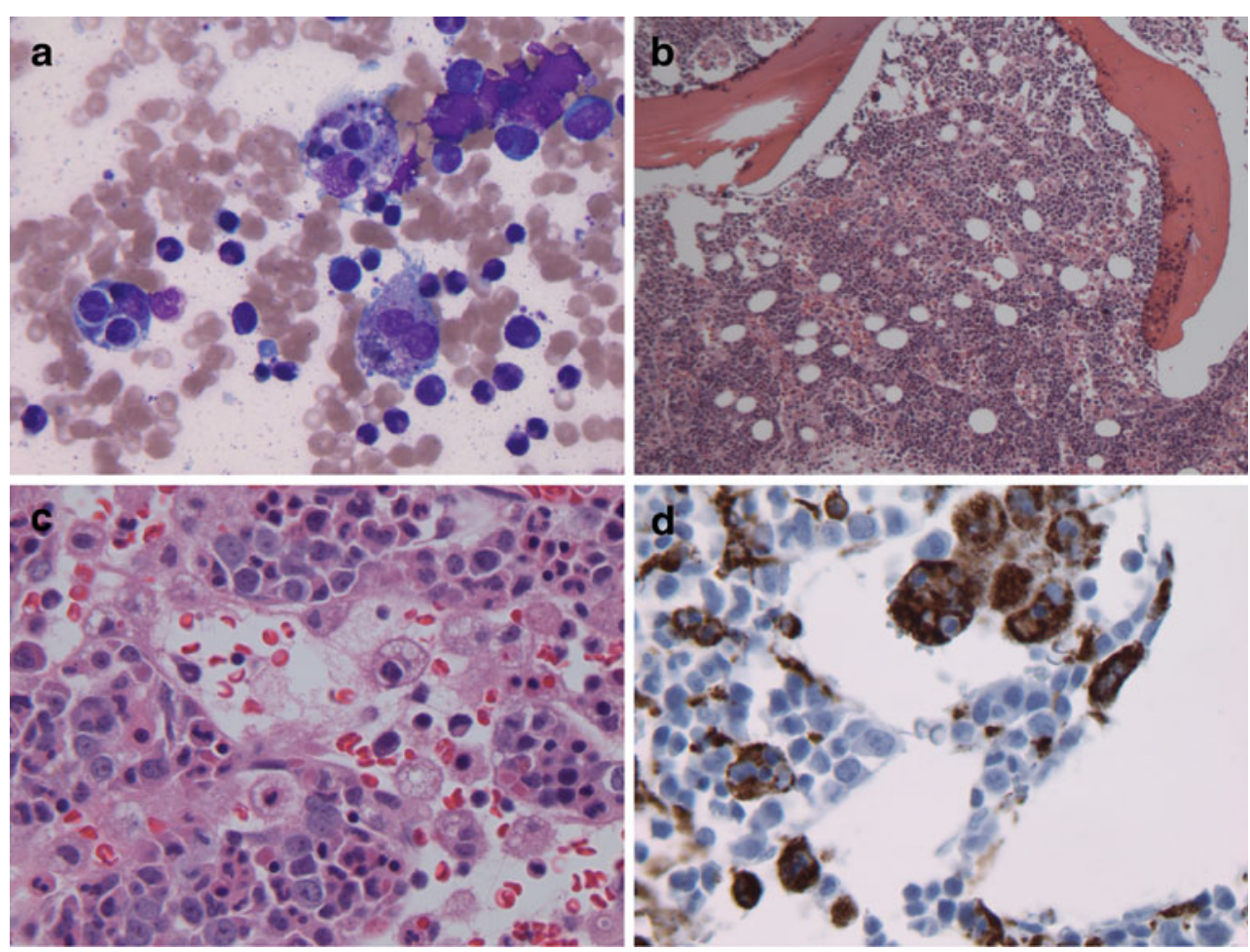

to the patient's influenza A (likely H1N1) infection. Though the patient met diagnostic criteria for JMML on the basis of monocytosis, elevated WBC, and circulating myeloid precursors it was felt that concurrent HLH and viral infection invalidated the applicability of these criteria in this case.

Unexpectedly, karyotyping by routine G-banding on the unstimulated bone marrow aspirate sample identified multiple clonal cytogenetic abnormalities: 46, XX, t(5;12)(q35;q24.1) [15]; 46, XX, t(5;12)(q35;q24.1), t(6;8)(p21;q24) [2]; and, 47, XXX, t(5;12)(q35;q24.1) [2]. Cells with normal 46, XX [1] karyotype were also present, confirming that the abnormal clones were not constitutional. The $t(5 ; 12)$ was present in the stemline clone with evolution of an additional $\mathrm{t}$ $(6 ; 8)$ in one clone and $\mathrm{a}+\mathrm{X}$ in another. Analysis of fluorescence in situ hybridization (FISH) for $P D G F R B$ at 5q31 q33 (Vysis LSI CSF1R Spectrum Orange/LSI D5S23: D5S721 Spectrum Green, Abbott Laboratories, Abbott Park, IL) did not demonstrate involvement of this locus. There was no evidence of the Philadelphia chromosome and molecular assays for $B C R-A B L 1$ fusion were negative. With the satisfaction of an additional diagnostic criterion, namely a clonal (non-recurrent) cytogenetic abnormality, a tentative diagnosis of JMML was made.

Additional testing was performed in an attempt to support the diagnosis of HLH and JMML. The patient's soluble interleukin 2 receptor level as measured by ELISA (Pierce Biotechnology Inc., Rockford IL, USA; courtesy of Dr. M. Abdelhaleem, Toronto, ON) was $32,400 \mathrm{U} / \mathrm{ml}$ (normal $=342-1,580 \mathrm{U} / \mathrm{mL}$ ). Perforin protein expression as a percentage of $\mathrm{CD} 56+$ cells was marginally reduced at $76.07 \%$ (normal $>80 \%$ ); the NK cell cytotoxicity assay was also marginally reduced at $15 \%$ (control 18-35; courtesy of Dr. M. Abdelhaleem, Toronto, ON) [20, 21]. Direct gene sequencing of PRF1 showed no evidence of mutation in the coding regions of exons 2 and 3 using a previously published method [22]; of note, a silent heterozygous polymorphism (900 T $>$ C) was detected. Serologic testing was positive for ANA, anti-RMP, ENA and anti-Sm but negative for anti-dsDNA, ANCA and rheumatoid factor. The patient's HbF level was not elevated $(<1.0 \%)$ and it was too late to assay for GM-CSF hypersensitivity in vitro.

The patient was started on treatment as per the HLH2004 protocol including 8 weeks of VP-16 $150 \mathrm{mg} / \mathrm{m}^{2}$, dexamethasone $10 \mathrm{mg} / \mathrm{m}^{2}$ for 2 weeks, and cyclosporin $6 \mathrm{mg} / \mathrm{kg}$ daily for 8 weeks [2]. Intrathecal therapy was deferred until later in the treatment course due to the patient's unstable status. Over the next month, the patient's condition improved steadily and she was extubated, the inotropes stopped, and Prisma hemofiltration was switched to intermittent hemodialysis. Nearly all signs and symptoms of HLH resolved and repeat bone marrow examination demonstrated no evidence of hemophagocytosis, histiocytosis or monocytosis; post-chemotherapy changes were present but there was no evidence of neoplasia; cytogenetic studies demonstrated a normal 46,XX karyotype. The only persistent abnormality was splenomegaly, which had decreased in size but remained mildly enlarged for age at 
$12.4 \mathrm{~cm}$. Treatment was stopped and the patient was discharged.

Approximately 1 month after discharge, the patient began to develop a number of tender, erythematous, indurated skin lesions on the left forearm and shoulder. Biopsy of these nodules revealed sheets of small- to medium-sized monomorphic cells with focal areas of necrosis. Initial immunostaining demonstrated strong CD45, CD4, and CD7 expression with variable CD3 positivity seen in approximately half of cells, suggesting a T-cell lymphoproliferative disorder. Additional marker studies demonstrated strong positivity for CD30 as well as cytoplasmic and nuclear ALK1, confirming the diagnosis of ALCL. The tumor had a high mitotic rate and MIB1 (Ki-67) staining demonstrated a proliferation index of nearly $100 \%$.

Staging bone marrow examination was performed immediately after diagnosis of ALCL. This sample is negative for ALCL by H\&E, CD30 and ALK1 stains as well as by G-banding karyotype. However, retrospective review of the patient's original bone marrow biopsy sample does demonstrate a low level of involvement by ALCL. Close re-inspection of the bone marrow aspirate identifies a few cells that fit the profile of ALCL, which had previously been labeled as atypical monocytes (Fig. 2a). Retrospective review of the H\&E-stained trephine biopsy also identifies rare hallmark cells (Fig. 2b). On immunostaining, these demonstrate cytoplasmic and nuclear positivity for ALK1 (Fig. 2c) with membrane and Golgi positivity for CD30 (Fig. 2d). Using these immunostains, marrow involvement appears to be minimal; affecting approximately $1-3 \%$ of cellularity and with diffuse single-cell positivity. Metaphase FISH was performed over previously G-banded cells from this original marrow sample using the Vysis LSI ALK Dual Color, Break Apart Rearrangement Probe (Abbott Laboratories). Analysis revealed that the $\mathrm{t}(5 ; 12)(35 ; \mathrm{q} 24.1)$, detected in all three abnormal clone lines was actually a three-way translocation: $\mathrm{t}(2 ; 5 ; 12)(\mathrm{p} 23 ; \mathrm{q} 35 ; \mathrm{q} 24.1)$.ish(5' ALK +;3'ALK;3',5'ALK-) likely resulting in fusion of $N P M-A L K$ on the $\operatorname{der}(5)$.

The patient was started on the Children's Oncology Group (COG) protocol ANHL0131 for advanced stage Anaplastic Large Cell Lymphoma with resolution of skin lesions. At the time of writing, the patient is 12 months into the treatment and continues to do well without any detectable evidence of HLH or ALCL.

\section{Discussion}

This case illustrates the diagnostic difficulties that can arise when attempting to determine the underlying etiology of HLH; the differential diagnosis is broad, it produces numerous symptoms that can mask other underlying conditions and it is relatively uncommon. The patient's history of preceding upper respiratory symptoms and positive influenza A serology initially pointed to influenza as the trigger of her HLH. However, viral upper respiratory tract infections in children are common and it is expected
Fig. 2 Bone marrow aspirate and trephine biopsy demonstrating ALCL. a Aspirate demonstrating a putative ALCL cell (May-Grünwald Giemsa). b Rare hallmark cells on the trephine biopsy. c Nuclear and cytoplasmic ALK1 immunostaining. d Membrane and Golgi CD30 immunostaining
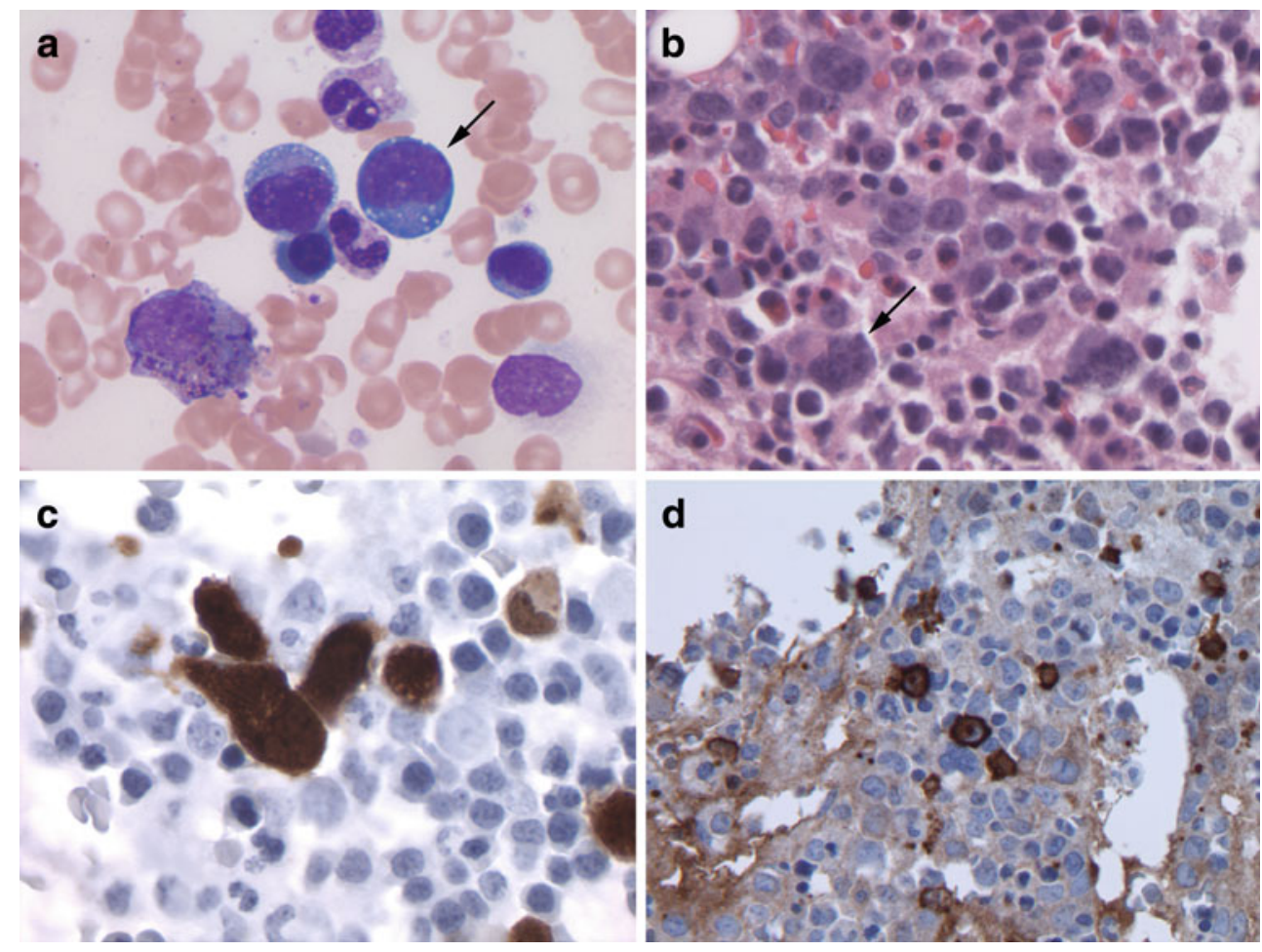


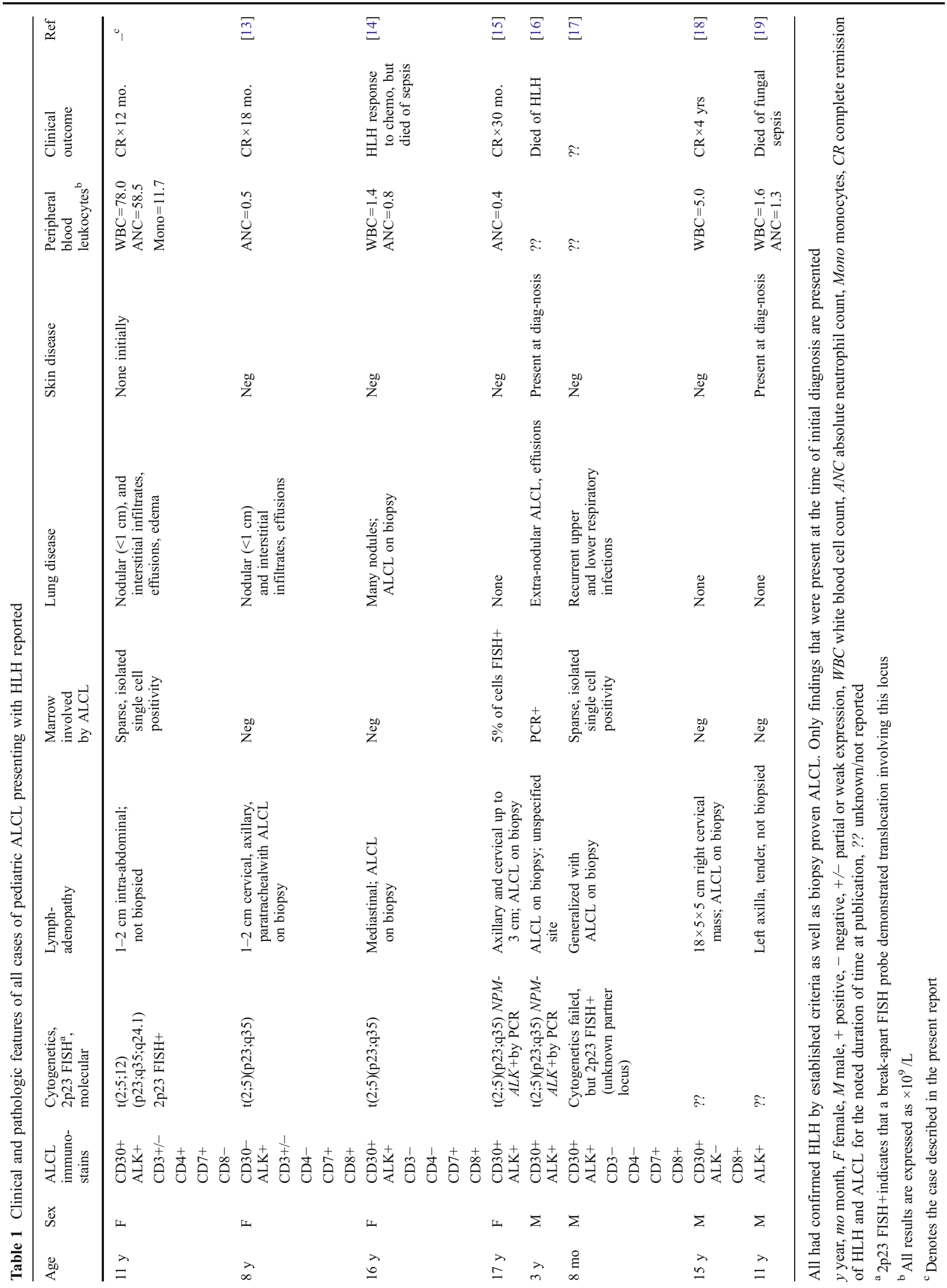


that many children or their guardians will provide a coincidental history of such an infection. Alternatively, it is possible that the combination of ALCL with a viral infection was the trigger for HLH. In either case, it is clear that other underlying causes of HLH must be sought, even when there is evidence of recent viral infection.

A unique feature of our case was the presence of a marked neutrophilic and monocytic leukocytosis which is atypical for both HLH and ALCL. Of cases reported in the literature where these two conditions occur together, ours is the only one associated with leukocytosis (Table 1). Together with the identification of a clonal cytogenetic abnormality this led to the incorrect initial diagnosis of JMML according to accepted WHO criteria [11]. It is important to note that this misdiagnosis was not due to overestimation of monocytes by misidentification of ALCL cells, though in retrospect some misidentification did occur. Immunostaining demonstrates minimal marrow involvement by ALCL (1-3\%) while monocytes account for a striking $24 \%$ of cellularity. The presence of a very pronounced monocytosis led to the much smaller proportion of ALCL cells being overlooked or dismissed as atypical monocytes. Similarly, retrospective review of the peripheral blood film also suggests that there was a minor population of ALCL cells whose presence was masked by monocytosis. GM-CSF hypersensitivity in vitro testing could not be performed, but even if it had been the presumably negative results would not have been able to exclude JMML [11]. Furthermore, an association between JMML and HLH has been recently reported [4] and the primary cytogenetic abnormality in our case, $\mathrm{t}(5 ; 12)(\mathrm{q} 35$; q24.1), has previously been associated with monocytic neoplasms. Greipp et al. describe the same $t(5 ; 12)(q 35$; q24.1) translocation in a patient with chronic myelomonocytic leukemia [23]. This group also describes a case of atypical chronic myelocytic disorder associated with monocytosis that had a similar translocation- $\mathrm{t}(5 ; 12)(\mathrm{q} 31 ; \mathrm{q} 24.1)$ [23]. The involvement of $12 \mathrm{q} 24.1$ is of particular interest because this locus is known to bear the most commonly mutated oncogene in JMML-PTPN11 (previously known as $S H P 2$ ), present in $35 \%$ of cases [11]. Unfortunately, it is not known if PTPN11 was involved in our case. The nonspecific nature of the current diagnostic criteria for JMML has been previously acknowledged and there are ongoing efforts to refine them [12].

In our case, the first feature to argue against JMML was the excellent response to HLH specific chemotherapy. JMML is notoriously difficult to manage and essentially resistant to all chemotherapy regimens [12]. The development of cutaneous lesions was not specific for either JMML or ALCL, both of which are frequently associated with extranodal tissue infiltration, particularly of the skin and lung $[6,11]$. However, it was much easier to identify ALCL on skin biopsy, then in the bone marrow, because of the uniform tumor cell population. The final factor to argue for a diagnosis of ALCL was the demonstration of cryptic $N P M-A L K$ fusion by FISH.

$\mathrm{HLH}$ associated with systemic ALK + ALCL is described in a small number of case reports of predominantly pediatric patients, which is not surprising given that these are both mainly diseases of childhood. Including the present case, there are a total of eight pediatric cases included for review with ages ranging from 8 months to 17 years and a proportionate male to female distribution (summarized in Table 1). Seven of the eight cases demonstrate an ALK + immunophenotype and six of these had cytogenetic or molecular studies performed that confirmed genetic $A L K$ rearrangement. Five of the seven ALK+ALCL cases specifically had a $\mathrm{t}(2 ; 5)(\mathrm{p} 23 ; \mathrm{q} 35)$ translocation and/or an NPM-ALK fusion product; in the other two cases the partner gene was not identified, but it is unlikely to be critical for the development of HLH.

There does not appear to be a correlation between increasing ALCL tumor burden and the risk of HLH. All of the cases had bone marrow examination, showing histiocytic proliferation with hemophagocytosis while only half of cases (4/8) demonstrated marrow involvement by ALCL. In those cases where marrow ALCL was present, three out of four cases had minimal involvement and the degree of involvement in the remaining case was not described. Thus, not only does routine morphologic assessment of the marrow have the potential to miss the diagnosis of ALCL, but it may also be masked by the more prominent HLH. Similarly, while all of the cases demonstrated lymphadenopathy, this was quite mild - typically in the range of 1$2 \mathrm{~cm}$, with the exception of the case of ALK- ALCL that presented with an $18 \times 5 \times 5 \mathrm{~cm}$ cervical mass. Mild lymphadenopathy may be overlooked under the circumstances because it can be caused directly by HLH. Of the six cases where a lymph node biopsy was performed, all six demonstrated involvement by ALCL-highlighting the importance of biopsy of this tissue. Thus, it appears that the occurrence of HLH does not depend on an advanced stage of ALCL or high tumor burden, making bone marrow biopsy poorly sensitive for disease and suggesting that lymph node biopsy should be undertaken even if enlargement is minimal.

Another diagnostic difficulty that may be encountered, particularly when tumor burden is low, is the inconsistent immunophenotype of ALCL. In our case and at least one other, ALCL was present as diffusely dispersed single-cells in the marrow without any evidence of tumor formation and the majority of cases demonstrated decreased or absent CD3 expression, which is known to be a frequent occurrence in ALCL [6]. Thus, screening H\&E and CD3/ CD20 immunostains on bone marrow samples can easily 
miss the diagnosis - as occurred in our case. Further, of seven cases where CD30 expression was tested, there was one case with absent expression of this marker. Similarly, ALK was negative in one of the eight cases, emphasizing the importance of assessing both CD30 and ALK immunostaining before involvement by ALCL can be ruled out.

A diagnostically useful recurring feature in HLH with ALCL appears to be extranodal involvement, particularly of the lung. This can manifest as pleural effusions, interstitial infiltrates, edema and/or parenchymal nodularity with at least four out of eight cases demonstrating these features. Lung involvement is not a typical feature of HLH [1], yet ALCL is well known to involve this extranodal site [6]. HLH due to a pulmonary infection or secondary lung infection due to neutropenia could produce some of these chest X-ray findings, but is unlikely to cause pulmonary nodules. Three of the eight cases demonstrated fine $(\leq 1 \mathrm{~cm})$ pulmonary nodularity, one of which was directly demonstrated to be caused by ALCL on biopsy. Though routine biopsy of these nodules would be difficult and possibly dangerous to the patient, the presence of lung nodules on imaging can be used as an indicator of the need for lymph node biopsy. Similarly, ALCL skin involvement is a very useful finding as HLH does not usually involve the skin and biopsy is easy and safe.

It is difficult to detect and correctly identify a hematolymphoid malignancy as the trigger of HLH in part because of overlapping clinico-pathologic features. Systemic ALCL is a particularly challenging diagnosis to make in this context because of its low tumor burden at presentation, and because if its immunophenotypic characteristics. The bone marrow is the most frequently biopsied site in HLH but is not sufficient to rule out systemic ALCL, or other lymphoproliferative disorders. The possibility of ALCL should always be considered in pediatric HLH, but clues that should heighten suspicion include nodular pulmonary infiltrates, skin involvement, other soft tissue involvement and/or lymphadenopathy.

Disclosures This publication was prepared without external funding. The authors declare that they have no conflict of interest.

\section{References}

1. Filipovich AH (2006) Hemophagocytic lymphohistiocytosis and related disorders. Curr Opin Allergy Clin Immunol 6:410-415

2. Henter JI, Horne A, Arico M, Egeler RM, Filipovich AH, Imashuku S, Ladisch S, McClain K, Webb D, Winiarski J, Janka G (2007) HLH-2004: diagnostic and therapeutic guidelines for hemophagocytic lymphohistiocytosis. Pediatr Blood Cancer 48:124-131

3. Janka GE (2007) Hemophagocytic syndromes. Blood Rev 21:245-253
4. Unal S, Cetin M, Kutlay NY, Elmas SA, Gumruk F, Tukun A, Tuncer M, Gurgey A (2010) Hemophagocytosis associated with leukemia: a striking association with juvenile myelomonocytic leukemia. Ann Hematol 89:359-364

5. Fornari A, Piva R, Chiarle R, Novero D, Inghirami G (2009) Anaplastic large cell lymphoma: one or more entities among Tcell lymphoma? Hematol Oncol 27:161-170

6. Delsol G, Falini B, Muller-Hermelink HK, Jaffe ES, Gascoyne RD, Stein H, Kinney MC (2008) Anaplastic large cell lymphoma (ALCL), ALK-positive. In: Swerdlow SH, Campo E, Harris NL, Jaffe ES, Pileri SA, Stein H, Thiele J, Vardiman JW (eds) WHO classification of tumors of haematopoietic and lymphoid tissues, 4th edn. IARC, Lyon, pp 312-316

7. Mann G, Gadner H (2008) Comment on HLH in a child with anaplastic large cell lymphoma (ALCL). J Pediatr Hematol/Oncol 30:721-722

8. Stein H, Foss HD, Durkop H, Marafioti T, Delsol G, Pulford K, Pileri S, Falini B (2000) CD30(+) anaplastic large cell lymphoma: a review of its histopathologic, genetic, and clinical features. Blood 96:3681-3695

9. Palmer RH, Vernersson E, Grabbe C, Hallberg B (2009) Anaplastic lymphoma kinase: signalling in development and disease. Biochem J 420:345-361

10. Morris SW, Kirstein MN, Valentine MB, Dittmer KG, Shapiro DN, Saltman DL, Look AT (1994) Fusion of a kinase gene, ALK, to a nucleolar protein gene, NPM, in non-Hodgkin's lymphoma. Science 263:1281-1284

11. Baumann I, Bennett JM, Niemeyer CM, Thiele J, Shannon K (2008) Juvenile myelomonocytic leukemia. In: Swerdlow SH, Campo E, Harris NL, Jaffe ES, Pileri SA, Stein H, Thiele J, Vardiman JW (eds) WHO classification of tumors of haematopoietic and lymphoid tissues, 4th edn. IARC, Lyon, pp 82 84

12. Chan RJ, Cooper T, Kratz CP, Weiss B, Loh ML (2009) Juvenile myelomonocytic leukemia: a report from the 2nd International JMML Symposium. Leuk Res 33:355-362

13. Blatt J, Weston B, Belhorn T, Hamrick H, Maia D (2002) Childhood non-Hodgkin lymphoma presenting as hemophagocytic syndrome. Pediatr Hematol Oncol 19:45-49

14. Sevilla DW, Choi JK, Gong JZ (2007) Mediastinal adenopathy, lung infiltrates, and hemophagocytosis: unusual manifestation of pediatric anaplastic large cell lymphoma: report of two cases. Am J Clin Pathol 127:458-464

15. Krenova Z, Sterba J, Blatny J, Kren L, Slany J (2007) A case of anaplastic large cell lymphoma-induced hemophagocytic lymphohistiocytosis in an adolescent female. Pediatr Blood Cancer 49:1056

16. Shimada A, Kato M, Tamura K, Hirato J, Kanegane H, Takechi Y, Park MJ, Sotomatsu M, Hatakeyama S, Hayashi Y (2008) Hemophagocytic lymphohistiocytosis associated with uncontrolled inflammatory cytokinemia and chemokinemia was caused by systemic anaplastic large cell lymphoma: a case report and review of the literature. J Pediatr Hematol/Oncol 30:785-787

17. Khor TS, Alessandri A, Jevon GP (2010) Infant anaplastic large cell lymphoma with hemophagocytic syndrome. Pediatr Dev Pathol 13:72-76

18. Sovinz P, Lackner H, Schwinger W, Benesch M, Urban C, Beham-Schmid C (2007) Anaplastic large cell lymphoma presenting as hemophagocytic syndrome in an adolescent. Pediatr Blood Cancer 49:1057

19. Burnham JM, Kreiger PA, Paessler M, Kersun LS, Cron RQ (2006) Picture of the month: anaplastic large cell lymphoma with hemophagocytic lymphohistiocytosis. Arch Pediatr Adolesc Med 160:1177-1179

20. Schneider EM, Lorenz I, Muller-Rosenberger M, Steinbach G, Kron M, Janka-Schaub GE (2002) Hemophagocytic lymphohis- 
tiocytosis is associated with deficiencies of cellular cytolysis but normal expression of transcripts relevant to killer-cell-induced apoptosis. Blood 100:2891-2898

21. Kogawa K, Lee SM, Villanueva J, Marmer D, Sumegi J, Filipovich AH (2002) Perforin expression in cytotoxic lymphocytes from patients with hemophagocytic lymphohistiocytosis and their family members. Blood 99:61-66
22. Stepp SE, Dufourcq-Lagelouse R, Le Deist F, Bhawan S, Certain S, Mathew PA, Henter JI, Bennett M, Fischer A, de Saint BG, Kumar V (1999) Perforin gene defects in familial hemophagocytic lymphohistiocytosis. Science 286:1957-1959

23. Greipp PT, Dewald GW, Tefferi A (2004) Prevalence, breakpoint distribution, and clinical correlates of $\mathrm{t}(5 ; 12)$. Cancer Genet Cytogenet 153:170-172 